\title{
The effects of Rotation on Wolf-Rayet Stars and on the Production of Primary Nitrogen by Intermediate Mass Stars
}

\author{
Georges Meynet ${ }^{1}$ and Max Pettini ${ }^{2}$ \\ 1) Geneva Observatory, CH-1290 Sauverny, Switzerland \\ 2) Institute of Astronomy, Madingley Road, Cambridge, CB3 OHA, UK
}

\begin{abstract}
We use the rotating stellar models described in the paper by A. Maeder \& G. Meynet in this volume to consider the effects of rotation on the evolution of the most massive stars into and during the Wolf-Rayet phase, and on the post-Main Sequence evolution of intermediate mass stars. The two main results of this discussion are the following. First, we show that rotating models are able to account for the observed properties of the Wolf-Rayet stellar populations at solar metallicity. Second, at low metallicities, the inclusion of stellar rotation in the calculation of chemical yields can lead to a longer time delay between the release of oxygen and nitrogen into the interstellar medium following an episode of star formation, since stars of lower masses (compared to non-rotating models) can synthesize primary N. Qualitatively, such an effect may be required to explain the relative abundances of $\mathrm{N}$ and $\mathrm{O}$ in extragalactic metal-poor environments, particularly at high redshifts.
\end{abstract}

\section{The Effects of Rotation on the Evolution into the Wolf-Rayet Phase}

As is recalled in the paper by P. Eenens (2004), Wolf-Rayet (WR) stars are the bare cores of initially massive stars, whose H-rich envelope has been removed by strong stellar winds or through Roche lobe overflow in a close binary system. Here we consider the following question: what are the effects of rotation on the evolution of massive single stars into the Wolf-Rayet phase? This subject has been discussed by Maeder (1987), Fliegner \& Langer (1995), Maeder $\&$ Meynet (2000) and Meynet (2000). We shall briefly summarise the main results presented in those papers and assess their importance in the framework of a new grid of massive star models at solar metallicity (Meynet \& Maeder in preparation).

As a preamble, let us reconsider the criteria which have been chosen to decide when a stellar model enters into the WR phase. Ideally, of course, the physics of the models should decide when the star is a WR star. However our poor knowledge of the physics involved, as well as the complexity of models coupling the stellar interiors to the winds, are such that this approach is not yet possible. Instead, it is necessary to adopt some empirical criteria for deciding when a star enters the WR phase. In this work the criteria are the following: the star is considered to be a WR star when its temperature is $\log T_{\text {eff }}>4.0$ and the mass fraction of hydrogen at the surface is $X_{S}<0.4$. Reasonable changes 
to these values (for instance adopting $X_{S}<0.3$ instead of 0.4 ) do not affect the results significantly.

Figure 1 shows the evolutionary tracks and the evolution of the structure in a non-rotating and a rotating $60 \mathrm{M}_{\odot}$ model. The rotating model has a timeaveraged equatorial velocity on the Main Sequence (MS) of about $190 \mathrm{~km} \mathrm{~s}^{-1}$, not far from the mean equatorial velocity observed for O-type stars. Inspection of Fig. 1, shows the following differences:

- The non-rotating $60 \mathrm{M}_{\odot}$ star goes through an intermediate Luminous Blue Variable (LBV) phase before becoming a WR star, while the rotating model skips the LBV phase and goes directly from the O-type star phase into the WR phase. One notes also that during the O-type star phase, the rotating track is bluer than its non-rotating counterpart. This is due to the diffusion of helium into the outer radiative zone (see Heger \& Langer 2000; Meynet \& Maeder 2000). On the other hand, the luminosity during the WC phase is not different between the rotating and the non-rotating model. Since these stars follow a mass-luminosity relation (Maeder 1987; Schaerer \& Maeder 1992), this implies that the masses of these two stars at this stage are about equal. At first sight this may be surprising. One expects the rotating star to lose more mass than its non-rotating counterpart. However, in this particular case there are some compensating effects. Indeed, the non-rotating model enters into the WR phase later, but becomes redder during the MS phase and then becomes a LBV star. During these two last phases, the star undergoes strong mass loss (see the left panels of Fig. 1). The rotating model, on the other hand, enters the WR phase at an earlier stage, reaching at the end an identical mass to the non-rotating model. In general, however, the final masses obtained from the rotating models are smaller than those obtained from the non-rotating ones, leading to lower luminosities during the WC phase.

- Focusing on the lower panels of Fig. 1, it can be realised that the nonrotating star enters in the WR phase with a mass of about $27 \mathrm{M}_{\odot}$. Nearly the whole $\mathrm{H}$-rich envelope has been removed by stellar winds during the previous phases (more precisely at the end of the MS and during the LBV phase). In this case, the main mechanism responsible for making the star a WR star is mass loss by stellar winds. For the rotating model, on the other hand, the entrance into the WR phase occurs at an earlier stage (although not at a smaller age!), while the star is still burning hydrogen in its core. The mass of the star at this stage is about $45 \mathrm{M}_{\odot}$ and an important $\mathrm{H}$-rich envelope is still surrounding the convective core. The main effect responsible for making the star a WR star in this second case is rotationally induced mixing (Maeder 1987; Fliegner \& Langer 1995, Meynet 2000).

These differences between the behaviours of the rotating and the nonrotating models have important consequences for the duration of the WR phase as a whole and for the lifetimes spent in the different WR subtypes, as can be appreciated from Fig. 2 . 

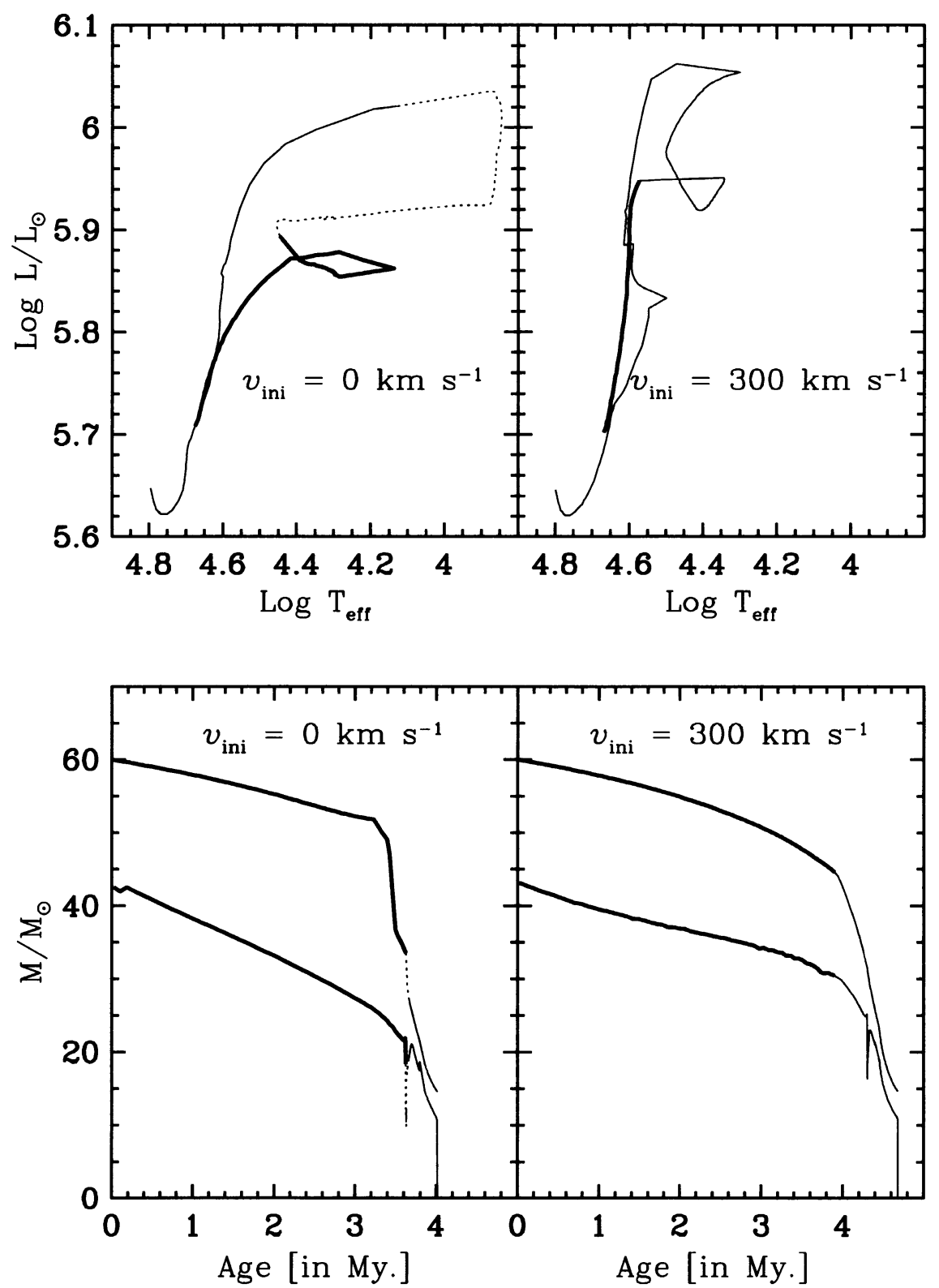

Figure 1. The two upper panels show the evolutionary tracks of a nonrotating and a rotating $60 \mathrm{M}_{\odot}$ stellar model at solar metallicity. The bold part of the track corresponds to the O-type star Main-Sequence phase; the dotted part show the intermediate phase, when present, between the $\mathrm{O}$-type star phase and the WR phase; the thin continuous line is the track during the WR phase. The two lower panels show the evolution as a function of time of the total mass and of the masses of the convective cores during the $\mathrm{H}-$ and the He-burning phases. 

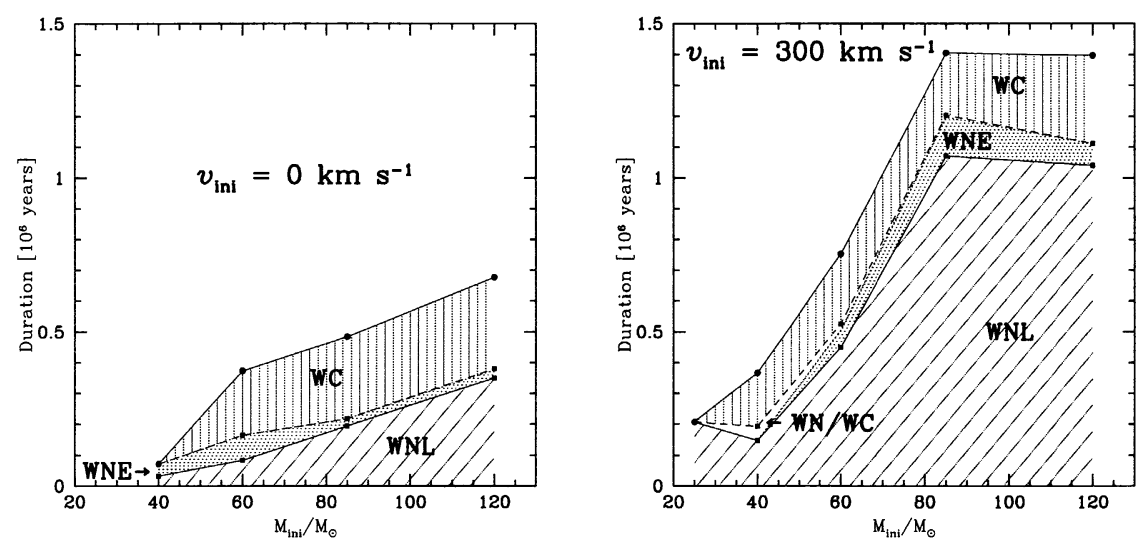

Figure 2. Durations of the WR phases and of the WR sub-phases for stars of various initial masses. The left panel shows the case for non-rotating models while the right panel corresponds to the case of rotating models.

- First, the WR lifetimes are enhanced. The typical enhancement for the 60 $\mathrm{M}_{\odot}$ model amounts to nearly a factor of 2 .

- Second, the WNL phase (the phase during which CNO processed material appears at the surface together with $\mathrm{H}$ ) is considerably lengthened. This is a natural consequence of the fact that, when the rotating stellar model enters the WR phase, it still has an important H-rich envelope. The WNL phase duration increases with the initial velocity. When the initial velocity passes from 300 to $500 \mathrm{~km} \mathrm{~s}^{-1}$ (or when the time-averaged equatorial velocity during the O-type phase goes from 190 to $330 \mathrm{~km} \mathrm{~s}^{-1}$ ), the WNL phase duration is increased by a factor of about 1.7. The durations of the WNE and WC phases are, on the other hand, affected much less (the WNE phase corresponds to the time when one sees at the stellar surface CNO processed material without hydrogen; during the WC phase, He-burning products such as carbon and oxygen appear at the surface).

- Thirdly, in the rotating stellar model a new phase of modest, but of nonnegligible duration, appears: the so-called transitional WN/WC phase. This phase is characterized by the simultaneous presence at the surface of both $\mathrm{H}-$ and $\mathrm{He}$-burning products (typically $\mathrm{N}$ and $\mathrm{C}$ or $\mathrm{Ne}$ ). The reason for this are the shallower chemical gradients which build up inside the rotating models. These shallower gradients inside the stars also produce a smoother evolution of the surface abundances as a function of time (or as a function of the remaining mass, see Fig. 7 in Maeder \& Meynet 2000). For a transitional WN/WC phase to occur, it is necessary to have - for a sufficiently long period-both a He-burning core and a CNO-enriched envelope. In the highest mass stars, mass loss removes too rapidly the $\mathrm{CNO}$-enriched envelope to allow a transitional WN/WC phase to appear. In the low mass range, the time spent in the WR phase is too short and the $\mathrm{H}$-rich envelope too extended to allow $\mathrm{He}$-burning products to diffuse up 
to the surface. Consequently, the transitional WN/WC phase only appears in the mass range between 30 and $60 \mathrm{M}_{\odot}$.

- Finally, the minimum mass for a star to become a WR star (through the single star channel) is lowered by rotation. In the present case, the minimum mass is reduced from about $37 \mathrm{M}_{\odot}$ for $v_{\text {ini }}=0 \mathrm{~km} \mathrm{~s}^{-1}$ to about $22 \mathrm{M}_{\odot}$ for $v_{\text {ini }}=300 \mathrm{~km} \mathrm{~s}^{-1}$.

From the above considerations, one can conclude that, compared to nonrotating models, stellar models including rotation will predict higher values of: (a) the numbers of WR stars relative to O-type stars, (b) the relative number of WN to WC stars, and (c) the relative number of transition WN/WC stars to WR stars. Are the observed values of these ratios in better agreement with the predictions of the rotating or non-rotating models? Clearly rotating models are in much better agreement.

However, before discussing further this conclusion, it is worthwhile recalling some of the difficulties faced by non-rotating stellar models. It was shown by Maeder \& Meynet (1994) that the relative numbers of WR and O-type stars observed in regions of different metallicities could be well reproduced by the stellar models, provided the mass loss rates were enhanced by about a factor of two during the MS and WNL phases. This is true not only for regions of constant star formation rate, but also for starburst regions (Schaerer et al. 2000). This enhancement appeared to be a reasonable assumption at the time of that study, given the uncertainties in the mass loss rates determinations available then. However, this solution is no long applicable today. Indeed it is now well established that the mass loss rates during the WR phase should be reduced by a factor of 2-3 owing to clumping effects in the wind (Nugis and Lamers 2001; Hamann et al. 1999). Thus we are left with the following problem: how can one account for the observed WR populations without increasing artificially the mass loss rates? The solution presumably lies in some physical process, not accounted for in the models of 1994, which would act as an enhancement of the mass loss rates as far as WR star formation is concerned.

Another difficulty faced by standard non-rotating models is the observed fraction of WR stars in the transition WN/WC phase. Although modest (a few percent), this fraction is much greater than predicted by non-rotating stellar models (with or without enhanced mass loss rates). This last discrepancy clearly points towards the need for an extra-mixing mechanism, as proposed by Langer (1991).

From our discussion above it is evident that rotation may help in solving these problems. Like enhanced mass loss rates, rotation lengthens the WR phase and decreases the minimum mass for a single star to go through a WR phase. Adopting the $\mathrm{O}$ and WR lifetimes obtained from the rotating models, the $\mathrm{WR} / \mathrm{O}, \mathrm{WN} / \mathrm{WC}$ and $(\mathrm{WN} / \mathrm{WC}) / \mathrm{WR}$ number ratios at solar metallicities are well reproduced (Meynet \& Maeder in preparation), while predictions for these same quantities obtained from non-rotating models are in clear disagreement with the observed values. Thus, one mechanism-rotational mixing-helps in solving both the problem of the WR/O number ratio and that of the transition WN/WC phase. It is somewhat encouraging to see that inclusion in stellar 
models of one observed characteristic of massive stars, namely their fast rotation, improves significantly the match of the models to reality.

We conclude this section on WR stars with a few additional remarks.

- For the moderate velocities considered here $\left(v_{\text {ini }}=300 \mathrm{~km} \mathrm{~s}^{-1}\right.$ on the ZAMS), the effects of wind anisotropies (Maeder \& Desjacques 2001) which are accounted for in the present models-play a modest role.

- Rotation may help in producing WR stars from single stars in metal poor environments, where the mass loss rates are expected to be much lower than at solar metallicity. This can result for at least two reasons. First, rotational diffusion can deplete the surface hydrogen abundance, making the star enter the WR phase well before mass loss has uncovered the core of the star. Second, since mass loss removes less angular momentum from the surface, the star can reach the break-up limit more easily. In this case, important mass loss rates ensue and this may help the star enter into the WR phase.

- As discussed by P. Eenens (2004), very little is known about the rotation of WR stars. The present models predict that the equatorial velocity is between 40 and $70 \mathrm{~km} \mathrm{~s}^{-1}$ during the WR phase. This corresponds to rotational periods of about 70 days during the WN phase and of a few days during the WC phase. A more complete discussion of this point will be found in the paper by Meynet \& Maeder (in preparation).

\section{Primary Nitrogen Production by Rotating Metal-Poor Intermediate-Mass Stars}

Primary nitrogen is produced by the transformation, through the CNO cycle in $\mathrm{H}$-burning zones, of carbon and oxygen synthesised by the star itself in $\mathrm{He}-$ burning regions. Nitrogen also has a secondary origin when the seed carbon and oxygen were already present in the star at the time of its formation. These two channels result in different behaviours of the abundance of $\mathrm{N}$ as a function of metallicity, as measured from a primary element such as oxygen. Primary $\mathrm{N}$ production is reflected by a constant $\mathrm{N} / \mathrm{O}$ ratio whereas, when secondary $\mathrm{N}$ takes over, the value of $\mathrm{N} / \mathrm{O}$ is expected to rise linearly with $\mathrm{O} / \mathrm{H}$. The existence of a plateau at $\log (\mathrm{N} / \mathrm{O}) \simeq-1.5$ in $\mathrm{H}$ II regions with $\log (\mathrm{O} / \mathrm{H}) \lesssim-4.0$ (that is, $\mathrm{O} / \mathrm{H} \lesssim 1 / 5$ solar) is generally interpreted as evidence for a dominant role of primary production of $\mathrm{N}$ at low metallicities (e.g. Edmunds \& Pagel 1978; Henry et al. 2000; see also Fig. 3).

There is less agreement, however, as to whether this primary nitrogen is produced by massive or intermediate-mass stars. The problem has attracted considerable attention in the last few years, not only because a knowledge of the yields of stars of different masses is central to chemical evolution models, but also because in principle in may be possible to use the $\mathrm{N} / \mathrm{O}$ ratio as a 'clock' to date the star formation activity in galaxies at different cosmological times (Edmunds \& Pagel 1978; Pettini, Lipman, \& Hunstead 1995; Pettini et al. 2002). The sensitivity of such a clock depends on the time delay between the release into the interstellar medium (ISM) of freshly synthesised $\mathrm{N}$ and $\mathrm{O}$. 


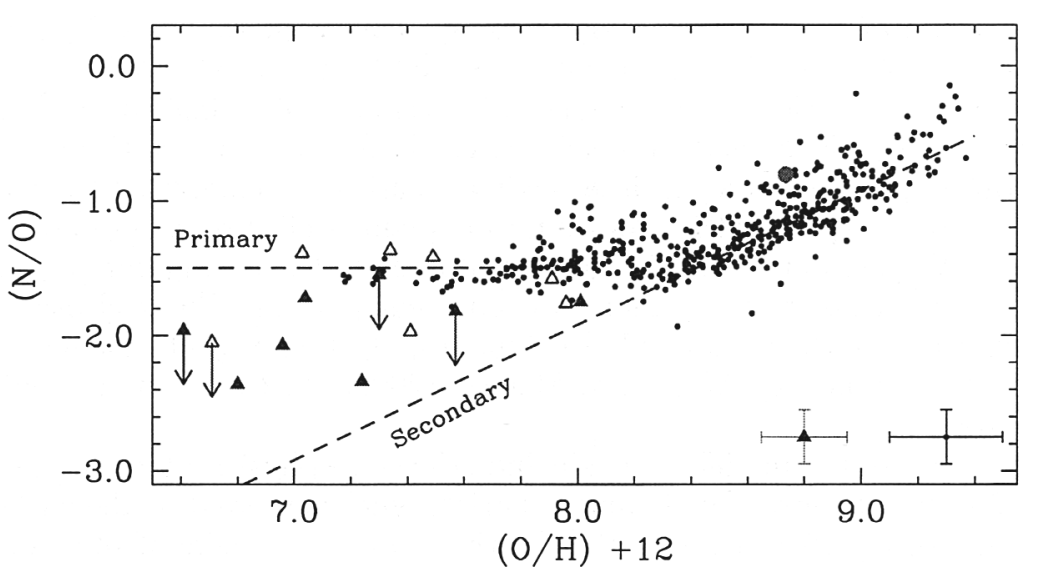

Figure 3. Abundances of $\mathrm{N}$ and $\mathrm{O}$ in extragalactic $\mathrm{H}$ II regions (small dots) and damped Lyman $\alpha$ systems (large triangles). Filled triangles denote DLAs where the abundance of $\mathrm{O}$ could be measured directly, while open triangles are cases where sulfur was used as a proxy for oxygen. The large dot corresponds to the solar abundances of $\mathrm{N}$ and $\mathrm{O}$ from the recent reappraisal by Holweger (2001). The dashed lines are approximate representations of the primary and secondary levels of $\mathrm{N}$ production. The error bars in the bottom right-hand corner give an indication of the typical uncertainties. More details regarding this figure and its interpretation may be found in Pettini et al. (2002).

Consider the limiting (and no doubt oversimplistic) case of a series of bursts of star formation separated by quiescent periods. Under these circumstances, the $\mathrm{N} / \mathrm{O}$ ratio is at first expected to decrease, as the $\mathrm{O}$ produced by the most massive stars which explode as supernovae is mixed with the ISM and the $\mathrm{O} / \mathrm{H}$ ratio increases accordingly. Only later will the N/O rise back to the primary plateau, as the lower mass stars which are the source of primary $\mathrm{N}$ evolve and release the products of their nucleosynthesis.

Clearly, no such behaviour would result if there is significant primary $\mathrm{N}$ production by massive stars. Thus, the relative uniformity of the $\mathrm{N} / \mathrm{O}$ ratios measured in nearby metal-poor galaxies led Izotov \& Thuan (1999) to propose that massive stars can perhaps produce significant amounts of primary $\mathrm{N}$. However, this uniformity could also be explained if these galaxies have not experienced much star formation in the recent past, as indicated by analyses of their stellar populations. In contrast, a wide range of values of $\mathrm{N} / \mathrm{O}$ is found in star-forming galaxies at intermediate redshifts (Contini et al. 2002) and in quasar absorption systems at high redshifts (Pettini et al. 2002; Prochaska et al. 2002). Even here, though, the interpretation is not straightforward. The problem is that the currently accepted value for the time delay in the release of primary $\mathrm{N}$ is $\Delta t \simeq 250 \mathrm{Myr}$, an interval which seems too short to explain the 
high proportion of galaxies and absorption systems with values of $\mathrm{N} / \mathrm{O}$ below the primary plateau (see Fig. 3), as discussed by Pettini et al. (2002).

The value $\Delta t \simeq 250 \mathrm{Myr}$ was deduced by Henry et al. (2000) from chemical evolution models which made use of the yields for primary $\mathrm{N}$ through 'Hot Bottom Burning' (see below) in intermediate mass stars computed by van den Hoek and Groenewegen (1997). As we now explain, stellar rotation may be the solution-at least in part-to this problem, if it shifts the production of primary $\mathrm{N}$ towards lower mass stars, with longer evolutionary times. The nucleosynthesis of $\mathrm{N}$ during the core $\mathrm{He}$-burning phase of metal-poor rotating intermediate-mass stars has been studied by Meynet \& Maeder (2001, 2002). Summarizing their main results:

1) Rotating stellar models which include the same physics which successfully accounts for: (a) the levels of $\mathrm{He}$ and $\mathrm{N}$ enrichments measured at the surface of stars in the Galaxy and in the SMC; (b) the relative numbers of blue and red supergiants in the SMC; and (c) the populations of WR stars at solar metallicity, naturally lead to the production of primary $\mathrm{N}$ by intermediate mass stars at very low metallicities (the metallicity considered in these models was 1/2000 of solar).

2) The process for this production of primary $N$ is different from, but not incompatible with, the classical scenario invoked up to now for intermediate mass stars, i.e. Hot Bottom Burning (HBB). The mechanism involving rotation produces primary $\mathrm{N}$ by diffusion of (primary) $\mathrm{C}$ and $\mathrm{O}$ from the He-burning core into the hydrogen burning shell. This mechanism occurs not only in intermediate mass stars but also, although with less efficiency, in massive stars ${ }^{1}$. The production of primary $\mathrm{N}$ by the HBB process occurs in intermediate mass stars only. After the core He-burning phase, the star evolves along the asymptotic giant branch. There, thermal instabilities drive thermal pulses which, in some circumstances, may inject $\mathrm{C}$ from the He-burning shell into the base of the convective envelope where the temperature may be sufficiently high for the CNO cycle to take place (see the review on AGB by Frost \& Lattanzio 1995). The quantities of primary nitrogen produced by this process remain difficult to predict (see Marigo 2001; Siess et al. 2002) and seem to depend heavily on the way mass loss and the third dredge-up mechanism are treated. Our models were computed up to the first 3-4 thermal pulses only. Since the HBB process is expected to occur at later stages, this means that in our models we did not account for any contribution from Hot Bottom Burning.

3) For average rotational velocities on the MS of the order of $230 \mathrm{~km} \mathrm{~s}^{-1}$, it was found that the most important contributors to the production of primary $\mathrm{N}$ are stars in the mass range between 3 and $5 \mathrm{M}_{\odot}$. The massive star contribution is, for this range of velocities, very modest.

4) The contribution of this process to primary $\mathrm{N}$ production is sufficiently efficient to explain a significant fraction of the primary $\mathrm{N}$ observed in low metallicity environments (Meynet \& Maeder 2002; Prantzos 2002; Carigi \& Pettini in this volume).

\footnotetext{
${ }^{1}$ In massive stars, the distance between the He-core and the $\mathrm{H}$-burning shell is greater than in intermediate mass stars; moreover the lifetimes are shorter thus, for a given initial velocity, the primary $\mathrm{N}$ production is much less efficient.
} 

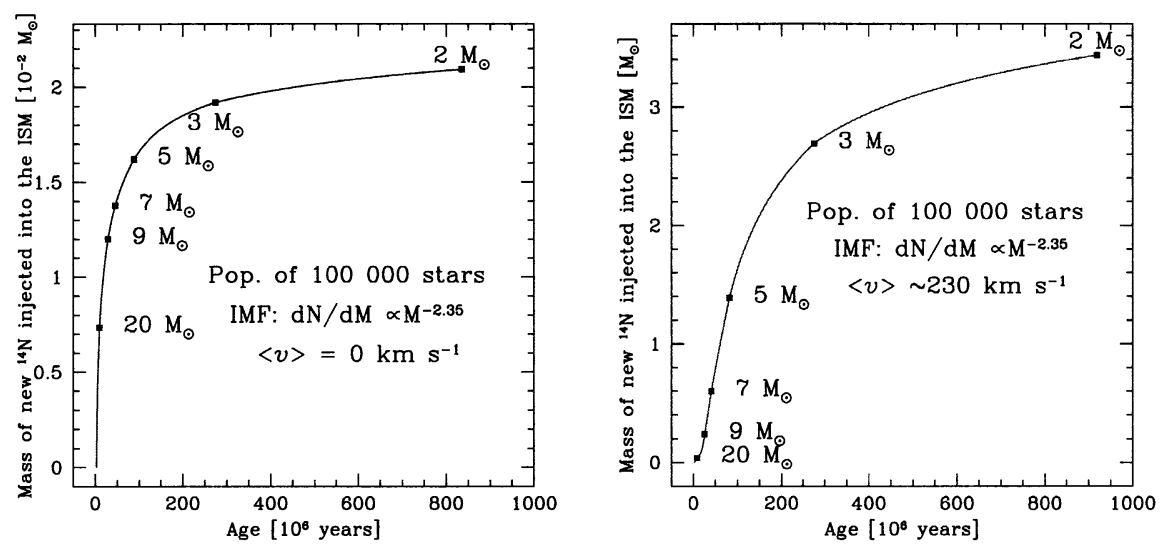

Figure 4. Quantities of newly synthesized nitrogen (in solar masses) injected into the interstellar medium by 100000 stars born at time $t=0$ with initial masses between 0.1 and $60 \mathrm{M}_{\odot}$. The stellar models have an initial mass fraction of heavy elements equal to 0.00001 (1/2000 the solar metallicity). The left panel shows the situation for non-rotating stellar models, while the right panel shows the case for rotating stellar models. Note that the vertical axis have different scales in the left and right panel. No hot bottom burning process is accounted for. The labels along the curves indicate when a star of a given initial mass contributes.

The effects of including stellar rotation in the calculations of the yields of primary $\mathrm{N}$ are illustrated in Fig. 4. Two main differences are noteworthy. First, the total quantity of primary $\mathrm{N}$ injected into the ISM by the rotating models is two orders of magnitude higher than that obtained with the non-rotating models. Second, $95 \%$ of the $N$ is ejected in a little more than $400 \mathrm{Myr}$ by the non-rotating models, whereas the time delay increases to $700 \mathrm{Myr}$ when rotation is taken into account. This larger value of $\Delta t$ is in good agreement with the empirical estimate derived by Pettini et al. (2002) from consideration of the fraction of damped Lyman alpha systems (DLAs) exhibiting values of (N/O) below the primary plateau at $\log (\mathrm{N} / \mathrm{O}) \simeq-1.5$ in Fig. 3 . In the framework of the model described above, these systems are interpreted as being observed in the period between the release of $\mathrm{O}$ by massive stars and of primary $\mathrm{N}$ by intermediate mass stars. By shifting the production of primary $\mathrm{N}$ further down the stellar initial mass function-and therefore increasing the time delay between the $\mathrm{O}$ and $\mathrm{N}$ enrichment of the ISM following an episode of star formation-stellar rotation naturally accounts for: (a) the otherwise surprisingly high fraction of DLAs with low values of $\mathrm{N} / \mathrm{O}$ in Fig. 3, and (b) the finding that most DLAs show little or no enhancement of the alpha-capture elements relative to Fe (e.g. Vladilo 2002). Taken together, these two considerations suggest similar timescales for the production of $\mathrm{N}$ and $\mathrm{Fe}$; the standard view is that approximately two thirds of the latter originates from Type Ia supernovae.

The agreement between the timescales predicted by the rotating models and those suggested by the DLA data is likely to be fortuitous. Observationally, a larger sample of measurements of $\mathrm{N}, \mathrm{O}$ and $\mathrm{Fe}$ in QSO absorbers is required for 
trustworthy statistics, while the models need to take into account a distribution of values of stellar rotation, as well as Hot Bottom Burning, in the calculation of the yields of primary N. They also need to be extended to a larger set of metallicities than considered so far. Nevertheless, the preliminary indications from Fig. 4 are that stellar rotation may well provide the 'missing clue' for the interpretation of $\mathrm{N}$ abundances.

Acknowledgments. G.M. expresses his deep gratitude to André Maeder for the very fruitful collaboration during the past years.

\section{References}

Contini, T., Treyer, M.A., Sullivan, M., Ellis, R.S. 2002, MNRAS 330, 75

Edmunds, M.G., Pagel, B.E.J. 1978, MNRAS 185, 77

Eenens, P., 2004, this volume

Fliegner, J., Langer, N. 1995. IAU Symp. 163, ed. K. A van der Hucht \& P.M. Williams, (Dordrecht: Kluwer), 326

Frost, C.A., Lattanzio, J.C. 1995. In Stellar Evolution: What Should Be Done", $32^{\text {nd }}$ Liège Int. Astroph. Coll., eds. Noels et al., 307

Hamann, W.R., Koesterke, L. 1999. IAU Coll. 169, ed. B. Wolf, O. Stahl, \& A.W. Fullerton, (Berlin: Springer). Also Lecture Notes in Physics, volume 523, 1999, p.239

Heger, A., Langer, N. 2000, ApJ 544, 1016

Henry, R.B.C., Edmunds, M.G., Koeppen, J. 2000, ApJ 541, 660

Holweger, H. 2001, in Solar and Galactic Composition, ed. R.F. Wimmer-Schweingruber (Berlin: Springer), 23

van den Hoek, L.B., Groenewegen, M.A.T. 1997, A\&ASS 123, 305

Izotov, Y.I., Thuan, T.X. 1999, ApJ 511, 639

Langer, N. 1991, A\&A 248, 531

Maeder, A. 1987, A\&A 178, 159

Maeder, A., Desjacques 2001, A\&A 372L, 9

Maeder, A. \& Meynet, G. 1994, A\&A 287, 803

Maeder, A. \& Meynet, G. 2000, ARA\&A, 38, 143

Marigo P. 1998, A\&A 340, 463

Marigo P. 2001, A\&A 370, 194

Meynet, G. 2000. In Massive Stellar Clusters, ASP Conf. Ser. 211, eds A. Lançon \& C. M. Boily, p. 105

Meynet, G., Maeder, A. 2000, A\&A 361, 101

Meynet, G., Maeder, A. 2002a, A\&A 381L, 25

Meynet, G., Maeder, A. 2002b, A\&A 390, 561

Nugis, T., Lamers, H.J.G.L.M. 2002, A\&A 389, 162

Pettini, M., Ellison, S.L., Bergeron, J., Petitjean, P. 2002, A\&A 391, 21

Pettini, M., Lipman, K., Hunstead, R.W 1995, ApJ 451, 100

Prochaska, J.X., Henry, R.B.C., O'Meara, J.M. et al. 2002, PASP, 114, 933

Schaerer, D., Maeder, A. 1992, A\&A 263, 129

Schaerer, D., Guseva, N.G., Izotov, Y.I., Thuan, T.X. 2000, A\&A 362, 53

Siess, L., Livio, M., Lattanzio, J. 2002, ApJ 570, 329

Vladilo, G. 2002, A\&A 391, 407 